\title{
A unique case of an alpha-fetoprotein-producing lung cancer with testicular metastasis
}

\author{
Jennifer M. Willder, MD; ${ }^{*}$ Andrew M. Thomson, MD; Kenneth M. Grigor, MD; Grahame Howard, MD; \\ Grant D. Stewart, $M D, P h D^{*}$
}

`Department of Urology, Western General Hospital, Edinburgh, Scotland; †Department of Pathology, Western General Hospital, Edinburgh, Scotland; ”Department of Oncology, Western General Hospital, Edinburgh, Scotland

Cite as: Can Urol Assoc J 2012;6(5):364-6. http://dx.doi.org/10.5489/cuaj.11088

\begin{abstract}
Alpha-fetoprotein (AFP)-producing primary lung tumours are rare; we present the first case of an AFP-producing lung tumour with metastasis to testes. The patient, a 72-year-old man, presented with a history of flu-like symptoms and abdominal pain. On examination he had a hard, tender left scrotal mass. Imaging showed a 4.4-cm right lower lobe lung mass and the serum-AFP was raised (1189 ng/mL). Left orchidectomy excised a necrotic tumour. Microscopy showed complete hemorrhagic infarction and immunohistochemistry showed a lack of staining for AFP. Serum-AFP rose 3 days post-orchidectomy to $1466 \mathrm{ng} / \mathrm{mL}$. The patient subsequently developed melaena and died. Autopsy revealed a $9 \times$ 5-cm necrotic right lower lobe lung tumour. Immunohistochemistry showed the tumour cells reacted with a pan-cytokeratin antibody and less than 5\% expressed AFP. Bilateral adrenal tumour deposits were also identified in addition to those in the bowel and spleen. The expression of AFP solely in the lung lesion and lack of expression in both testes, together with a rise in serum-AFP post-orchidectomy and the bilateral adrenal metastases, is overwhelming evidence for the reversal of the usual situation: a poorly differentiated AFP-secreting metastatic lung adenocarcinoma.
\end{abstract}

\section{Introduction}

Alpha-fetoprotein (AFP) is an important diagnostic tumour marker for hepatocellular carcinoma (HCC) and germ cell tumours (GCT). ${ }^{1}$ AFP-producing primary lung tumours are rare; therefore, an elevated serum-AFP in conjunction with a lung neoplasm is most likely to represent a metastasis. We present a case of an AFP-secreting metastatic lung neoplasm.

\section{Case report}

A 72-year-old man presented with flu-like symptoms and abdominal pain. His medical history included hypercholesterolaemia, hypertension, chronic obstructive airways dis- ease and alcohol excess. His drug history included lisinopril, simvastatin and amitryptiline. He had a 60-pack/year smoking history and was a retired coal miner.

On examination, he was hypotensive $(100 / 50 \mathrm{mmHg})$ and tachycardic (pulse 120 bpm). His abdomen was soft, but tender in the suprapubic region; the bowel sounds were normal. He had a hard, tender left scrotal mass. Initial investigations revealed acute renal failure (urea $17.2 \mathrm{mmol} / \mathrm{L}$; creatinine $327 \mu \mathrm{mol} / \mathrm{L}$ ) and a macrocytic anemia (Hb $91 \mathrm{~g} / \mathrm{L} ; \mathrm{MCV}$ $107 \mathrm{fL})$.

A computed tomography angiogram revealed a suspicious mass at the right lung base. Further imaging showed a 4.4-cm right lower lobe lung mass and a 3-cm mass contiguous with extensive right hilar lymph node enlargement. Bilateral enlarged adrenal glands, with cortical enhancement, and a thickened segment of small bowel, not seen on the initial scan, were also identified. Testicular ultrasound scan showed a $6 \times 4 \times 5-\mathrm{cm}$ heterogeneous left scrotal mass. In an assessment of serum tumour markers, AFP was raised at $1189 \mathrm{ng} / \mathrm{mL}$ (lactate dehydrogenase and $\beta$-human chorionic gonadotrophin [ $\beta$-hCG] were normal).

A provisional diagnosis of an AFP-secreting testicular neoplasm with pulmonary metastases was made. Although unusual in a 72-year-old, this diagnosis offered the possibility of a cure.

Left orchidectomy excised a $4 \times 4 \times 7$-cm hemorrhagic and necrotic tumour. Microscopy showed complete hemorrhagic infarction with peripheral fibrosis and attendant inflammation. The tumour had not breached the tunica albuginea or involved the rete testes or epididymis. Vacuolated cells were identified within the seminiferous tubules raising the possibility of co-existing intra-tubular germ cell neoplasia-unspecified. These features were consistent with a primary testicular tumour, which had undergone hemorrhagic infarction.

The patient subsequently started a course of carboplatin and etoposide chemotherapy. However, 3 days later he developed melena. Attempts at resuscitation were unsuccessful and he died. 


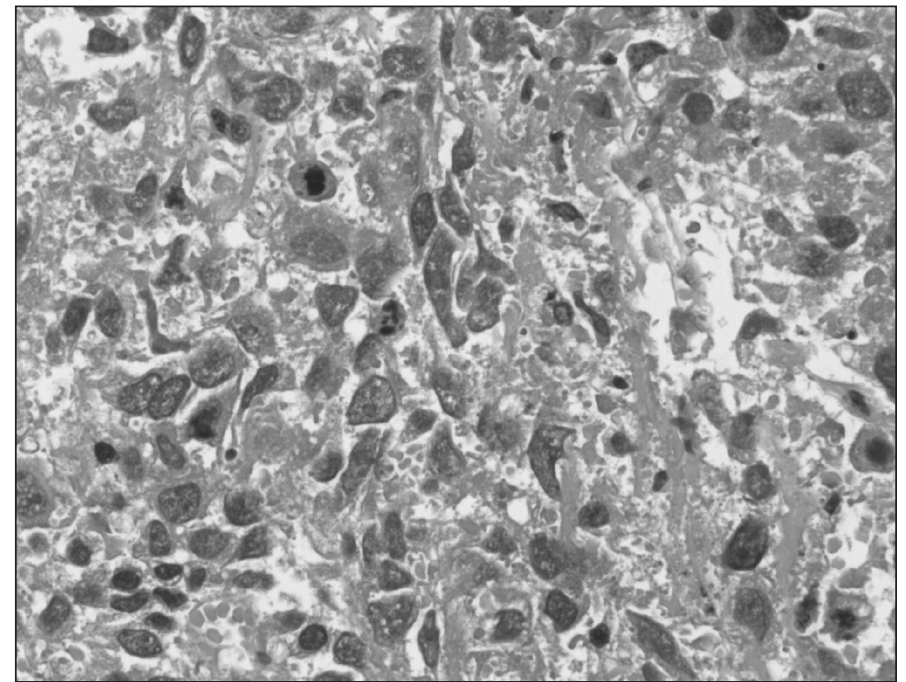

Fig. 1a. Representative photomicrograph of pleomorphic tumour in the right lung (haematoxylin and eosin; magnification $\times 400$ ).

An autopsy showed a $9 \times 5$-cm necrotic right lower lobe lung tumour. Microscopy showed a poorly cohesive, extensively necrotic, pleomorphic neoplasm (Fig. 1a). The tumour cells had a high nucleus-to-cytoplasmic ratio, coarse chromatin and, in a high percentage of cells, a single eosinophilic nucleolus; mitotic figures were readily identified. Tumour replaced and extended beyond a lymph node at the main bronchus.

Immunohistochemical analyses of the lung lesion showed the tumour cells reacted with a pan-cytokeratin (CK) antibody and less than 5\% expressed AFP (Fig. 1b). There was no evidence of CK7, thyroid-transcription factor-1, CEA, synaptophysin, OCT 3/4, CD30, $\beta$-hCG or CD117 expression. This profile is not typical of an embryonal carcinoma or a primary lung tumour, but could be consistent with a GCT with yolk sac-like features.

A tumour nodule within the jejunal mesentery had eroded into the bowel lumen, and was associated with distal gastrointestinal hemorrhage (the cause of the melena). Microscopy showed a glandular neoplasm with a similar immunoprofile to the lung tumour. Both adrenals were replaced by adenocarcinoma and the tumour was also present in the spleen. There was no evidence of tumour in the stomach or pancreas.

The original left orchidectomy specimen was examined further. Immunocytochemistry did not detect placental alkaline phosphatase or OCT 3/4 expression, indicating that the vacuolated cells within the seminiferous tubules were likely due to tubular atrophy. Immunohistochemical analyses of the right testes showed simple atrophy. Importantly, neither the left or right testicle expressed AFP. This suggests the testicular lesion was almost certainly a necrotic metastasis, substantiated by a rise in the serum-AFP level to $1466 \mathrm{ng} / \mathrm{mL} 3$ days post-orchidectomy. The most likely diagnosis is that of a

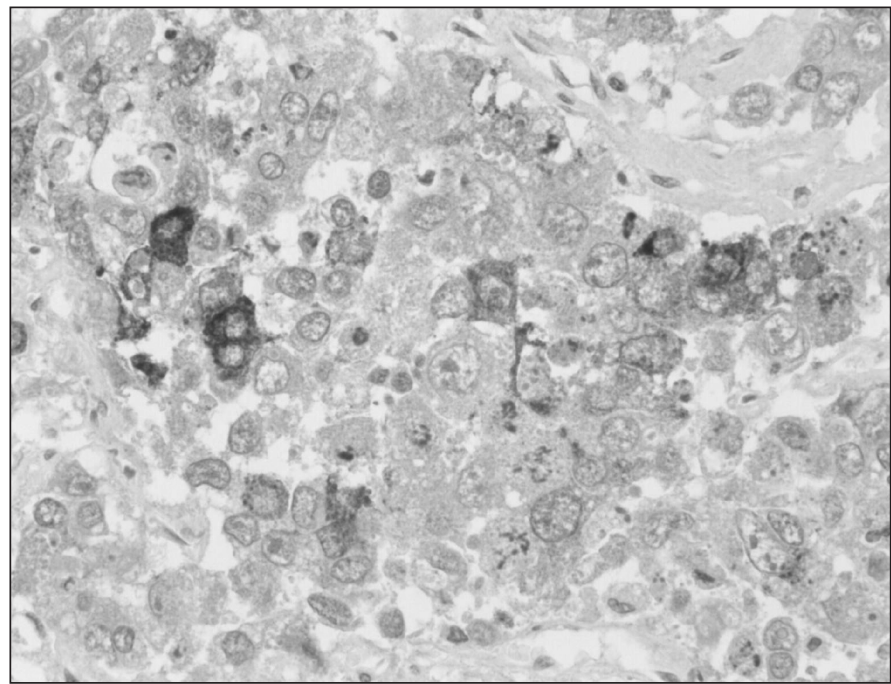

Fig. 1b. Immunohistochemical analysis of alpha-fetoprotein expression by the tumour cells (magnification $\times 400$ ).

poorly differentiated AFP-secreting metastatic lung adenocarcinoma.

\section{Discussion}

AFP is an oncofetal antigen with an important diagnostic role in primary liver tumours and GCTs. ${ }^{1-3}$ There is evidence gastric and pancreatic carcinomas may also produce AFP. 4,5 Reports of primary AFP-secreting lung tumours are rare. ${ }^{6-8}$

Lung metastases from a primary liver tumour or GCT can produce AFP. AFP levels can be elevated in patients with primary lung carcinoma associated with liver metastases; it is believed that regenerating hepatocytes surrounding the metastatic tumour cells are the source of AFP. ${ }^{9-10}$ Reports of AFP-producing primary lung cancer in the absence of liver metastases also exist. ${ }^{6-8}$ To our knowledge, this is the only reported case of an AFP-producing lung cancer, without liver involvement, associated with testicular metastasis.

The transcriptional and epigenetic changes responsible for the rare expression of AFP in primary lung neoplasms are unclear; it has been proposed that cell context may facilitate this process. One theory invokes the shared embryological origin of the lung, liver, pancreas and stomach and suggests that a common ontological heritage may partly explain "inappropriate" AFP-expression by tumours arising from these sites. ${ }^{1}$

In this case, the serum-AFP level was persistently elevated after orchidectomy, possibly caused by metastatic disease. It highlights that tumour markers can be difficult to interpret; the normal range does not have a normal distribution and the "normal" level for some patients (without an obvious underlying cause) is beyond the laboratory range. Furthermore, certain conditions and drugs can increase serum-AFP levels, specifically alkylating agents. This emphasizes that tumour 
Willder et al.

markers are not perfect indicators and must be interpreted within the clinical setting and context of multiple investigative parameters.

This case was reviewed by a specialist uro-pathologist; in conjunction with the multidisciplinary team, it was felt that the findings of the original left orchidectomy were sufficient to support the diagnosis of a metastatic AFP-secreting tumour with testicular origin. The fatal gastro-intestinal hemorrhage was most likely precipitated by chemotherapy resulting in rapid necrosis of the jejunal metastasis. Spontaneous rupture of metastases resulting in major hemorrhage can occur prior to chemotherapy, although this is rare. ${ }^{11}$ This case report highlights the importance of having a definitive pathological diagnosis prior to the initiation of chemotherapy.

\section{Conclusion}

This case report illustrates a reversal from the usual situation. Initially it was assumed that the lung lesion was metastatic from an AFP-secreting testicular tumour. Although there will always be a small degree of conjecture, the expression of AFP solely in the lung lesion and lack of expression in both testes, together with the rise in serum-AFP post orchidectomy and the bilateral adrenal metastases, which are typical for lung tumours, make the unusual situation of a primary AFPsecreting lung lesion with testicular metastases the mostly likely diagnosis. Thus, a primary lung neoplasm should be considered when a lesion is present within the lung fields and the serum-AFP is elevated.
Competing interests: None declared.

This paper has been peer-reviewed.

\section{References}

1. Gitlin D, Perricelli A, Gittin GM. Synthesis of -fetoprotein by liver, yolk sac, and gastrointestinal tract of the human conceptus. Cancer Res 1972;32:979-82.

2. O'Conor GT, Tatarinov YS, Abelev GI, et al. A collaborative study for the evaluation of a serologic test for primary liver cancer. Cancer 1970;25:1091-8. http://dx.doi.org/10.1002/10970142(197005)25:5<1091::AID-CNCR2820250514>3.0.C0;2-P

3. Masopust J, Kithier K, Rad J, et al. Occurrence of fetoprotein in patients with neoplasms and non-neoplastic diseases. Int J Cancer 1968;3:364-73. http://dx.doi.org/10.1002/iic.2910030306

4. Mclntire KR, Waldmann TA, Moertel CG, et al. Serum alpha-fetoprotein in patients with neoplasms of the gastrointestinal tract. Cancer Res 1975;35:991-6.

5. Kozower M, Fawaz KA, Miller HM, et al. Positive alpha-fetoglobulin in a case of gastric carcinoma. N Engl J Med 1971;285:1059-60. http://dx.doi.org/10.1056/NEJM197111042851906

6. Hiroshima K, lyoda A, Toyozaki T, et al. Alpha-fetoprotein-producing lung carcinoma: report of three cases. Pathol Int 2002;52:46-53. htrp://dx.doi.org/10.1046/j.1440-1827.2002.01311.x

7. Okunaka T, Kato H, Konaka C, et al. Primary lung cancer producing alpha-fetoprotein. Ann Thorac Surg 1992;53:151-2. http://dx.doi.org/10.1016/0003-4975(92)90778-3

8. Yamagata $T$, Yamagata $Y$, Nakanishi $M$, et al. A case of primary lung cancer producing alpha-fetoprotein. Can Respir J 2004;11:504-6.

9. Corlin RF, Tompkins RK. Serum alpha 1 -fetoglobulin in a patient with hepatic metastases from bronchogenic carcinoma. Am J Dig Dis 1972;17:553-5. http://dx.doi.org/10.1007/BF02231214

10. Tsung SH. Alpha-feto protein in lung cancer metastatic to the liver. Arch Pathol 1975;99:267-9.

11. Moore K, Imbeault A, Roy G, et al. Massive hemorrhage from spontaneous rupture of a retroperitoneal lymph node in patient with metastatic mixed germ cell tumor. Urology 2009;76:159-61. hittp://dx.doi. org/10.1016/i.urology.2009.10.001

Correspondence: Dr. Grant D. Stewart, Clinical Lecturer and Specialist Registrar in Urological Surgery, Department of Urology, Western General Hospital, Edinburgh, EH4 2XU; grant.stewart@ed.ac.uk 